Walter Bruno Berg

(Freiburg im Breisgau)

\title{
PASTOR S. OBLIGADO: TRADICIONES DE BUENOS AIRES. FUNKTION UND GESTALT EINER PERUANISCHEN GATTUNG AM CONO SUR
}

I.

\section{ZUR REZEPTION DER PERUANISCHEN TRADICIÓN}

Die inter-amerikanische Rezeption der in Peru entstandenen Erzähl-Gattung tradición gehört zu den noch wenig erforschten Gegenständen der an Rezeptionsphänomenen überaus reichen Fin de siècle-Epoche. Dank der editorischen und hermeneutischen Bemühungen namhafter Kritiker, vornehmlich peruanischer Nationalität ${ }^{\prime}$, wurde die Gründer-Figur selbst - Ricardo Palma - zwar bereits seit geraumer Zeit aus der Sekundärposition eines peruanischen Schul-Autors erlöst und in den Rang eines unbestrittenen Klassikers ${ }^{2}$ erhoben. Das Interesse der Kritiker bleibt jedoch - ganz im Einklang mit der traditionellen Genie-Ästhetik - autorengebunden. ${ }^{3}$ Es richtet sich kaum auf die als Epigonen betrachteten übrigen Vertreter der Gattung, sei es in Peru selbst, sei es in Bolivien, in Uruguay oder in Argentinien. Der Argentinier Pastor S. Obligado, mit dem ich mich im Folgenden beschäftigen will, wurde von der heutzutage als offiziell geltenden Literaturgeschichte so gut wie totgeschwiegen. ${ }^{4}$ Selbst für die Beschaffungspolitik der Biblioteca Nacional scheint er kaum eine Rolle gespielt zu haben. Sie verfügt über keine historische Ausgabe seiner Werke, sondern nur über eine

I Augusto Tamayo Vargas, Alberto Escobar, Miguel Oviedo, Julio Ortega, cf. die ausführliche Bibliographie bei Ricardo Palma (1993: 635-658).

2 Cf. jetzt auch die Ausgabe der Tradiciones peruanas in der spanischen Klassiker-Reihe Cátedra, besorgt von Carlos Villanes Cairo (1994).

3 Nur unter zwei Gesichtspunkten fand die Gattungsfrage in der Palma-Forschung bisher Beachtung: Zum einen hinsichtlich der von Palma selbst stammenden metaphorischen Gattungsdefinition - auf die weiter unten zurückzukommen sein wird -, zum anderen hinsichtlich einer durch die "critique génétique geleiteten Fragestellung nach der formengeschichtlichen Entstehung der Gattung als Befreiung aus den Prämissen der romantischen Ästhetik.

4 Es wurde ihm nicht einmal die Ehre zuteil, wie sein Zeitgenosse, der in den ersten Jahrzehnten dieses Jahrhunderts hochangesehene Manuel Gálvez, vom hohen Ross des zeitgenössischen Bewusstseins herab als Traditionalist gebrandmarkt zu werden. - Zu Gálvez cf. die Einleitung von David Lagmanovich zu Ricardo Szmetan (1994); siehe dazu meine Rezension (1996: 115-117). 
schmale Auswahl aus dem Jahre 1955. Die plakative Rezeption einer auch in ihrem Heimatland keineswegs unumstrittenen ${ }^{5}$ - speruanischen Gattung durch Obligado entsprach im liberal und modernistisch geprägten Fin de siècle-Kontext der argentinischen Hauptstadt, wo maßgebliche Intellektuelle im ideologischen Abwehrkampf gegen die andrängenden Massen der Einwanderer seit Neuestem außerdem mit der Konstruktion des rautochthonen« Mythos der argentinidad beschäftigt waren, offenbar einer Position der diskursiven Ort-Losigkeit, die von der offiziellen Kritik nicht anders als mit der Geste des Vergessens bestraft werden konnte. Schon 1897, ein Jahr vor Erscheinen der Tradiciones de Buenos Aires, bedurfte der Autor offenbar eines Fürsprechers. So erscheint in der Ausgabe vom 18. September 1897 der Tageszeitung La Nación eine diesbezügliche »Carta-Prólogo«, unterzeichnet von Ricardo Palma. Der in La Nación veröffentlichte Text ist identisch mit dem Vorwort der in Buchform erschienenen Originalausgabe.

Ricardo Palma ist also der Autor eines Vorworts zur >Vierten Serie` der Tradiciones de Buenos Aires. Mithin lässt er keinen Zweifel zu, dass er selbst, wie er ausdrücklich unterstreicht ${ }^{7}$, zu den begeisterten Lesern Obligados gehört. Die Rezeption ist demnach keine einseitige, sondern verläuft in doppelter Richtung. Palma scheint von der Lektüre so angetan, dass er sich herausgefordert fühlt, erneut zu grundsätzlichen Fragen der von Obligado und von ihm selbst praktizierten Gattung Stellung zu nehmen. Ich möchte zunächst einige Punkte aus dieser Stellungnahme herausgreifen und komme dann zur Lektüre von Obligado selbst.

\section{II. \\ RICARDO PALMAS VORWORT ZU DEN \\ TRADICIONES DE BUENOS AIRES VON PASTOR S. OBLIGADO}

Palmas Vorwort zu Obligado ${ }^{8}$ gehört zu den interessantesten theoretischen Texten des peruanischen tradicionista. Im Zentrum steht - einmal mehr die Frage nach einer zureichenden Definition der Gattung tradición. An an-

\footnotetext{
5 Exemplarisch sind in dieser Hinsicht die Polemiken von José Santos Chocanos.

6 Cf. Walter Bruno Berg / Markus Klaus Schäffauer (1999).

7 Cf. Ricardo Palma (1898: VII).

8 Verweise auf dieses Vorwort werden im Weiteren unter der Verwendung der Sigle CP sowie der entsprechenden Seite im fortlaufenden Text angegeben.
} 
derer Stelle habe ich bereits auf die Zwei- bzw. Vieldeutigkeit der bekannten Definition der tradición als "género ancilar de la historia « ${ }^{9}$ hingewiesen. Auch am Ende des Vorworts zu Obligado spielt Palma auf diese Formulierung an und scheint zunächst die traditionelle Gattungstrennung von Geschichtsschreibung und Fiktion ohne Wenn und Aber zu akzeptieren:

[...] siempre he reconocido que la tradición puede ser una de las fuentes auxiliares de la Historia, pero se me atraganta de que ella alcance á ser la historia misma. $(\mathrm{CP}, \mathrm{XI})^{10}$

Einige Zeilen weiter heißt es zum gleichen Thema:

Para el que sepa ó alcance á leer en la piedra, como en un documento, no es la Tradición la que le habrá servido de gran cosa para reconstruir la Historia. (Ibid.)

Die Rede ist abermals von der Arbeit des Historikers im Gegensatz zu der des tradicionista. Die Position von Palma lässt an Deutlichkeit nichts zu wünschen übrig. Man wird sie als >historistisch bezeichnen dürfen: Die Objektivität der Geschichte liegt im Know how - in Händen - des Historikers.

Schauen wir jedoch auf die ersten Abschnitte des Vorworts, so stoßen wir auf Formulierungen, die mit den zitierten nicht mehr unbedingt in Übereinstimmung zu bringen sind. Der Leser sieht sich einem wahren $F u$ rioso von sprachlichen Zweideutigkeiten und humoristischen Wendungen gegenüber, in dem das breite Reservoir populärer Periphrasen, Sprichwörter und Sentenzen, über welche die spanische Sprache verfügt, in einem auch für Palma ungewohnten Maße aktiviert wird. Wer hier spricht, ist offenbar nicht der Historizist, sondern der selbstbewusste Schriftsteller, oder sagen wir Sprach->Künstler :

[...] ocurrióme pensar que era hasta obra de patriotismo popularizar los recuerdos del pasado, y que tal fruto no podía obtenerse empleando el estilo severo del historiador, estilo que hace bostezar á los indoctos. (CP, VIII)

9 Cf. Ricardo Palma (1977: XIX). Zu meinen eigenen Überlegungen cf. Walter Bruno Berg (1998).

10 Wir behalten die Orthographie der Originalausgabe bei. - Der zitierte Satz ist eine Replik auf eine im vorausgehenden Abschnitt zitierte Definition des argentinischen Autors Joaquín V. González: "La Tradición es la Historia de los pueblos que no tienen Historia« (ibid.). "Historia« heißt in diesem Kontext, so jedenfalls interpretiert Palma die Äußerung von González, soviel wie »Geschichtsschreibung «, nicht etwa »Geschichte«. 
»Popularizar los recuerdos del pasado« - auch der tradicionista beschäftigt sich mit der Geschichte bzw. der Geschichtsschreibung. Aber >auf seine Weise « und demzufolge sanders als der professionelle Historiker. Davon, dass beide in einem hierarchischen Verhältnis zueinander stünden, dass der tradicionista lediglich $>$ Hilfsdienste leistete im Hinblick auf die ernsthafte Geschichtsschreibung, ist nicht mehr die Rede. Eher umgekehrt: Gemessen an den Zielen, die sich der tradicionista selbst setzt, macht er seine Sache ganz offensichtlich besser als der Historiker.

Um welche Arbeit, um welches Ziel - welches officium - jedoch geht es dem tradicionista? Wir müssen die Frage zunächst noch zurückstellen, denn Palma beantwortet sie - wenn überhaupt - nur indirekt. Was er zunächst einmal unterstreicht, ist etwas anderes. Nicht das Worumwillen, die Intention, der tradición scheint ihm wichtig, sondern ihr Wie, ihre literarische Form. Ohne die adäquate literarische Form ist der Inhalt nämlich nichtig:

El suceso aislado, por interesante y singular que sea, se parece á una jóven vestida de trapillo. $(\mathrm{CP}, \mathrm{X})$

Einige Zeilen weiter behauptet er sogar, die Form erschaffe den Inhalt:

Quien consagra sus ratos á borronear Tradiciones, debe tener lo que se llama la gracia del barbero, gracia que estriba en sacar patilla donde no hay pelo. (CP, X$\mathrm{XI})$

Der tradicionista muss die Kunst des Barbiers beherrschen, Koteletten hervorzuzaubern, wo kein Haar mehr wächst. Der tradicionista ist mithin Schriftsteller, schöpferischer Schriftsteller; daran besteht kein Zweifel.

Doch nun zurück zum Problem des Worumwillen, dem Ziel, dem Gegenstand der tradiciones. »[...] ocurrióme pensar que era hasta obra de patriotismo popularizar los recuerdos del pasado«, so hatte es oben geheißen. Das Ziel der neuen Gattung ist demnach ein doppeltes: ein politisches einerseits, ein - sagen wir - >ästhetisches` andererseits. Hinsichtlich des ersteren spricht Palma von "patriotismo«, von »sentimiento de americanismo « $(\mathrm{CP}, \mathrm{X})$, sogar von »ilustración«. Hinsichtlich des letzteren dagegen benutzt er das Schlüsselwort »popularizar«. Die neue Gattungsästhetik entspricht also einem Programm von Populär-Literatur - so scheint es jedenfalls:

El pueblo es como los niños, que tragan, y hasta con deleite, la píldora plateada. $(\mathrm{CP}, \mathrm{IX})$ 
Doch so einfach geht die Rechnung nicht auf. Die Formel lautete ja »popularizar los recuerdos del pasado«, nicht »popularizar la literatura«. Das Attribut des >Populären < bezieht sich Palma zufolge auf das erwähnte politisch-patriotische Ziel der tradiciones, nicht aber auf ihre literarische Form. Was die letztere angeht, so scheint es, als ob die Attribute >literarisch und >populär fast synonym gebraucht würden. Nur vor diesem Hintergrund erklärt sich die Emphase, mit der Palma im nächsten Abschnitt die Metapher des »platear píldoras « $(\mathrm{CP}, \mathrm{X})$ ironisch expandiert:

Bien haya, repito, la hora en que me vino en mientes el platear píldoras, dárselas á tragar al pueblo, sin andarme en chupaderitos ni con escrúpulos de monja boba. Algo, y aún algos, de mentira, y tal cual dósis de verdad, por infinitesimal ú homeopática que ella sea, muchísimo de esmero y cumplimiento en el lenguaje, y cata la receta para escribir Tradiciones. (Ibid.)

Genau 75 Jahre vor dem Erscheinen von Jacques Derridas Dissémination erinnert Palma daran, dass literarische Praxis ein gefährliches Geschäft ist. Literatur ist »píldora«; »pharmakon«, hieß dies bei Plato; »Arznei« also, eine Substanz von prinzipiell und grundsätzlich doppelter, ja zweideutiger Wirkung. Die Eigenschaften von Wahrheit und Lüge, Heilmittel oder Gift liegen eng beieinander. Ihre Unterscheidung sinkt herab zur Frage der richtigen Dosierung. Natürlich gehört die tradición zur Gattung der heilbringenden Substanzen, doch Palma sieht sich genötigt, die entgegengesetzte Eigenschaft - ihre Wirkung als Gift - explizit zu negieren:

Tengo conciencia de que no he propinado veneno, sinó pócima saludable para ilustración y entretenimiento del pueblo, amén de que es eminentemente sujestiva la indole literaria de esa clase de escritos. (Ibid.)

"¿No opina Vd. como yó, Doctor Obligado" ${ }^{11}$ « (ibid.), fügt er anspielungsreich hinzu. Auch Obligado ist somit aufgenommen in die Zunft dieser literarischen Wunder-Doktoren... Soviel zu Palma, doch nun zu Obligado selbst.

11 Die Anrede fällt aus dem Rahmen. "A mi amigo el doctor don Pastor S. Obligado«, heißt die Widmung des Vorworts. Die verschiedenen Anreden im Text sind: »mi querido Don Pastor« (p. VII), »amigo Pastor« (p. VIII), »mi querido señor Obligado« (p. XII). 
III.

PALMA UND DER DiSKURS DER VIRTUALITÄT

Der Diskurs der Fülle und der des Mangels - »el discurso de la abundancia y el discurso de la carencia $\ll^{12}$ - sind Julio Ortega zufolge die beiden maßgeblichen Identitätsdiskurse, mit denen lateinamerikanische Intellektuelle im 19. Jahrhundert die historische Realität ihrer Länder zu fassen versuchten. Es handelt sich um zwei Diskurse, deren Entstehung weit in die Kolonialzeit zurückverweist, die nun jedoch unter den historischen Bedingungen nationaler Unabhängigkeit erneut zur Disposition gestellt sind. Noch einen dritten, nicht minder dominierenden Diskurs glaubt Ortega ausfindig machen zu können, denjenigen der Virtualität:

[El] discurso [...] de lo virtual, que se propone, promete o asume un programa y un proyecto de acciones y realizaciones, para un futuro hecho de la diferencia heterogénea de lo latinoamericano $[\ldots]{ }^{13}$

Ausführlich dargestellt hat Ortega die Kategorien in einem programmatischen Artikel der von ihm selbst besorgten Studienausgabe der Tradiciones Peruanas in der Colección Archivos mit dem Titel »Las Tradiciones Peruanas y el proceso cultural del XIX hispanoamericano«. In der Tat: Vor dem Hintergrund der als >metahistorische` Ordnungsprinzipien aufgefassten Kategorien »Fülle«, »Mangel« und »Virtualität« tritt die epochale Bedeutung der Tradiciones nunmehr deutlich zutage. Diese lässt sich dahingehend zusammenfassen, dass bei Palma alle drei Kategorien gewissermaßen synchron bzw. gleichzeitig aktualisiert werden: Im Vordergrund steht zweifellos der satirische Mangel-Diskurs, die bereits von Mariátegui gegenüber der zeitgenössischen Kritik ins Feld geführte aufklärerisch-republikanische Akzentuierung der Tradiciones, die es verbietet, das Werk als Aushängeschild des politischen und ideologischen Konservativismus abzustempeln. Auch der Fülle-Diskurs ist bei Palma repräsentiert, allerdings in einer Form, von der uns das eben besprochene Vorwort bereits einen Vorge-

12 Julio Ortega (1993: 409).

13 Julio Ortega (1993: 410). Trotz der Dreizahl wäre es falsch, Ortegas Schema shegelianisch zu verstehen. Die Kategorien, obwohl - zumal die beiden ersteren - durchaus dualistisch und oppositionell konzipiert, implizieren keine geschichtsphilosophische Hypothese oder Teleologie, nicht einmal im strikten Sinne - eine Chronologie. Alle drei Diskurse finden sich vielmehr sowohl in ein und derselben Epoche als auch mitunter bei ein und demselben Autor. Es handelt sich um Perspektiven, Modellvorstellungen, unter denen die Welt gesehen wird - "grandes modelos de representación del mundo americano« (1993: 409). 
schmack geliefert hat. Wie eine Generation vor ihm Altamirano in Mexiko ist auch Palma zutiefst davon überzeugt, dass die Literatur nicht etwa als das Resultat des gesellschaftlichen Fortschritts zu betrachten sei, sondern vielmehr als eine seiner Ursachen. ${ }^{14}$ Der Schriftsteller wird damit zum Hüter und Bewahrer eines kulturellen Erbes, das bei Palma freilich keineswegs in den Dienst einer monolithischen Nationalideologie ${ }^{15}$ bzw. der Affirmation einer oligarchischen Elitekultur ${ }^{16}$ gestellt wird, sondern vielmehr in den Dienst eines grundsätzlich multiperspektivischen Modells, dessen Angelpunkt die Einbeziehung der gesamten Breite der populären, oral tradierten Episteme der peruanischen Nationalkultur ist. Der prinzipiell kritische Akzent dieser Aufwertung der Populär-Kultur liegt auf der Hand:

Lo popular es una forma de humor pero también un modo de evaluar y preservar la información; una vivacidad material, inmediata, que pervive a pesar de las crisis y de los códigos, como un centro de desplazamiento, con su propia legitimidad, sabiduría y capacidad de vida. ${ }^{17}$

Der humoristische Polyperspektivismus ist dann auch das entscheidende, zukunftsweisende und - in der Diktion Ortegas - >virtuelle Element der Tradiciones. Ohne das Schlagwort selbst zu nennen, interpretiert Ortega Palma in dieser Hinsicht als einen originären Post-Modernen avant la lettre:

Con los materiales de la Tradición, la «tradición» palmista es una verdadera deconstrucción de lo construido, de lo dado e inculcado. ${ }^{18}$

IV.

PASTOR S. OBLIGADO: »FIERAS HUMANAS «

Damit komme ich - schließlich und endlich - zu Obligado. Ich möchte der Frage nachgehen, ob und in welcher Hinsicht Obligados Texte dem eben skizzierten Gattungsmodell Tradiciones konform sind. Aufgrund der gebotenen Kürze kann ich die Frage nur anhand eines einzigen Textes verfol-

\footnotetext{
Julio Ortega (1993: 419).

Diese findet sich zum Beispiel im Werk des eben bereits erwähnten Mexikaners Altamirano.

16 So der Grundzug der »sátira limeña«, deren poetischer Wortführer der Satiriker Felipe Pardo y Aliaga (1806-1868) war, cf. Julio Ortega (1993: 430-431).

17 Julio Ortega (1993: 429).

18 Ibid.
} 
gen. Es handelt sich um die Erzählung »Fieras humanas«; sie stammt aus der von Palma eingeleiteten `Vierten Serieく.

Im Zentrum der tradición steht die den Warhafftige[n] Historien Einer Wunderbaren Schiffahrt (1599) des deutschen Söldners Ulrich Schmidel (1510-81) entnommene Geschichte der »Maldonado«. Schmidel gehört zu den ersten Chronisten der Eroberung und Besiedelung der Rio-de-la-PlataLänder. Ein immer noch gebräuchlicher Ortsname hat die Erinnerung an die Geschichte der Maldonado bis in die Gegenwart wach gehalten. »Maldonado - la mal abandonada « (TBA, 167) ${ }^{19}$, so deutet der Autor den Namen, ist eine Frau im Tross des spanischen Konquistadors Francisco Ruiz Galán. Vom Hunger getrieben verlässt sie eines Tages unerlaubter Weise das Lager der Soldaten. Als Ruiz Galán davon erfährt, lässt er sie zur Strafe in der Wildnis aussetzen. Als es Abend wird, sucht Maldonado Unterschlupf in einer Höhle. Plötzlich sieht sie sich einer leibhaftigen $»$ Löwin $\ll{ }^{20}$ gegenüber und fällt vor Schreck ohnmächtig zu Boden. Als sie aufwacht, stellt sie fest, dass die »Löwin«, statt sie anzugreifen, nach Hilfe sucht, denn sie ist trächtig und liegt in Wehen. Tatkräftig unterstützt durch Maldonado bringt sie anschließend zwei kleine »Löwenkinder« zur Welt. In den folgenden Tagen wird Maldonado nicht nur in die »Löwenfamilie« aufgenommen, sondern darüber hinaus durch die »Löwin « mit frisch gerissenem Fleisch versorgt. Eines Tages wird sie von Indios, die in der Nähe leben, aufgefunden und durch Heirat in deren Gemeinschaft aufgenommen. Einige Zeit später kommt es zu einem Zusammenstoß zwischen den Indios und dem Trupp der spanischen Konquistadoren. Maldonado wird gefunden. Die Strafe, die Ruiz Galán nun für sie ausgedacht hat, ist härter als die erste. Man fesselt sie an einen Baum, um sie von wilden Tieren zerreißen zu lassen. Die Tiere erscheinen, unter ihnen auch die menschenfreundliche »Löwin«. Als die Spanier zurückkommen, um den Vollzug der Exekution zu kontrollieren, sehen sie, dass die »Löwin « ihre Menschen-Freundin erfolgreich gegen ihre tierischen Artgenossen verteidigt. ${ }^{21}$ Nun scheinen auch die Spanier gerührt und geben der Frau die Freiheit.

19 Verweise auf diese Textausgabe werden im Weiteren unter Verwendung der Sigle TBA sowie der entsprechenden Seite im fortlaufenden Text angegeben.

20 Tatsächlich handelt es sich um einen Puma; es sei hier daran erinnert, dass dieser im amerikanischen Spanisch als »león « bezeichnet wird.

21 Siehe Abbildung. Die zahlreichen Illustrationen der Originalausgabe sind ausschließlich zeitgenössischer Provenienz. Der biedermeierlich-idyllisierende Grundzug, der die meisten von ihnen auszeichnet, entspricht dem Geschmack des 19. Jahrhunderts und findet sich auch in der hier wiederge- 


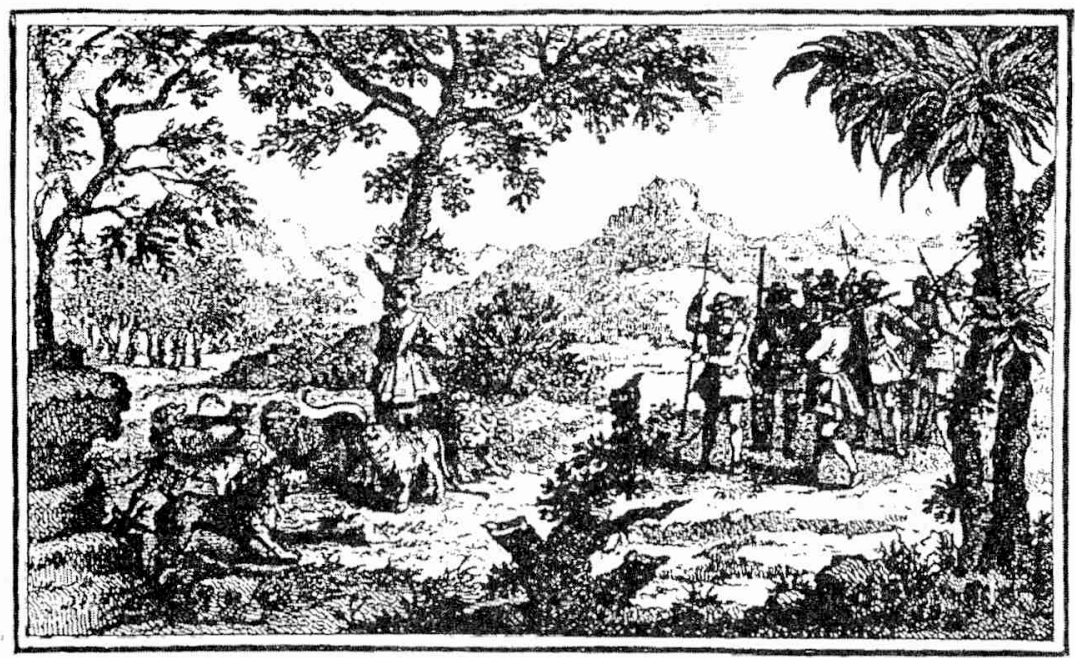

La Maldonado salvada por la Leona

(Tradicith del aüo 1534)

Abbildung aus: Pastor S. Obligado, Tradiciones de Buenos Aires

Die Geschichte der Maldonado ist nicht identisch mit der tradición als ganzer - weder quantitativ noch evaluativ, noch textuell. Sie wird zunächst in Form einer mit Ironiesignalen durchsetzten Nacherzählung dargeboten. Die Ironiesignale verweisen einerseits auf den Status des Textes als einer Nacherzählung, mithin auf seinen Quasi-Zitatcharakter; andererseits mahnen sie den Leser zur Vorsicht gegenüber der Wertung, die der Legende als solcher innewohnt. Letztere braucht, wie wir sehen werden, mit der allgemeinen Wertung der tradición nicht unbedingt übereinzustimmen. Der Rest der Geschichte - beginnend mit der Schilderung des Lebens der Maldonado im Schoße der »Löwenfamilie « - wird dagegen in Form eines wörtlichen Zitats aus Schmidels Warhafftiger Historie dargeboten.

gebenen Maldonado-Illustration: Unversehrt, mit weitausladendem Faltenrock, tiefausgeschnittener Bluse und eleganter Hochschlagfrisur ist Maldonado an einen Baum gefesselt, umringt von einer Schar großer Tiere, die den Trupp der bewaffneten Spanier in Schach halten. Die Szenerie entspricht ganz und gar der Sinngebung des Textes, nicht jedoch dem antispanischen Zungenschlag der berühmten Kupferstiche (1590-1634) des Theodor de Bry (1528-1598), wie in der Diskussion des Vortrags gemutmaßt wurde. 
Abgesehen von ihrer textuellen Differenzierung sind die beiden Teile der Geschichte durch zwei ausgedehnte Exkurse unterbrochen. Vom Textumfang her gesehen betragen sie ein Vielfaches der Maldonado-Geschichte. Die Exkurse ihrerseits gliedern sich in vier thematisch, aber auch textuell klar differenzierte Teile. Der erste Exkurs steht in der Mitte der beiden Teile der Maldonado-Geschichte. Seine Funktion besteht darin, mit biblischen und mythologischen Beispielen die der Legende immanente Evaluation, das heißt die These von der angeblichen Sanftmut der wilden Tiere, zu unterstreichen. Die übrigen drei Exkurse sind der Maldonado-Geschichte angehängt - so scheint es zunächst, denn diese endet ja mit der Befreiung Maldonados. In einem kurzen Epilog kommt Obligado jedoch am Ende noch einmal auf die Legende zurück und weist darauf hin, dass die Geschichte Maldonados dank der Namensgebung bis in die Gegenwart hinein im kollektiven Gedächtnis der Argentinier verwurzelt ist. Der allgemeine Aufbau der tradición erinnert somit an eine bekannte musikalische Form. Bezeichnet man mit $>\mathrm{A}<$ die Maldonado-Geschichte, mit $>\mathrm{B}<$ und $>\mathrm{C}<$ die Exkurse, so erhalten wir in der Tat ein Rondo: A - B - A - C - A. Anders als im klassischen Rondo liegt das thematische Gewicht des Textes jedoch nicht im A-Teil, sondern in Teil C. Er ist auch quantitativ gesehen der wichtigste, denn er enthält nicht weniger als drei Exkurse. Hier - also in Teil C - wird das eigentliche, durch den Titel vorgegebene Thema der tradición explizit behandelt: »fieras humanas«. Damit werden die übrigen Teile des Textes jedoch nicht etwa zu minderer Bedeutung verurteilt. Was der Verweis auf die musikalische Form vielmehr deutlich machen soll, ist die grundsätzliche Vielstimmigkeit des Textes, ein Bedeutungsaufbau des Ganzen also weniger in Form einer Hierarchie, bei welcher die einzelnen Teile einer einheitlichen Gesamtbedeutung untergeordnet sind, als vielmehr in Form eines Aggregats, bei welchem die Einzelteile ihr semantisches Eigengewicht behalten und sich zueinander im Verhältnis gegenseitiger Spannung befinden.

Warum der Titel »Fieras humanas «? Die drei Exkurse von Teil C geben eine Antwort. Das Anschauungsmaterial für die eigentlichen »fieras« - die eigentlichen »wilden«, oder, wie wir im Deutschen sagen: »Raubtiere« liefert nicht die Zoologie, sondern die Geschichte. Der erste Exkurs des CTeils schildert entsprechend einer der bekanntesten Gräueltaten lateinamerikanischer Geschichte, die grausam-groteske Hinrichtung Túpac Amarus II. und seiner acht Gefolgsleute auf der Plaza de Armas von Cuzco am 18. 
Mai 1781. Sein historisches Gewicht erhält der Exkurs u. a. dadurch, dass er - parallel zum zweiten Teil der Maldonado-Geschichte - als wörtliches Zitat einer zeitgenössischen Quelle präsentiert wird.

Doch die Gräuel der Geschichte, so unterstreicht der Autor, sind kein Privileg spanischer Konquistadoren:

[...] así como la virtud no tiene pátria, ménos la tiene el crímen [...] (TBA, 171).

Inhalt des zweiten Exkurses ist dementsprechend eine lange Liste historischer Grausamkeiten aus Europa, vornehmlich aus dem Land, das sich gerne als die Wiege der aufgeklärten, modernen Humanität bezeichnet: Frankreich.

Der dritte - und damit letzte - Exkurs des C-Teils verweist thematisch auf den allerersten Exkurs ${ }^{22}$ (der zwischen die beiden Teile der MaldonadoGeschichte eingefügt ist). Wiederum geht es um Beispiele von >Humanität< aus dem Tierreich. Sie sind nunmehr jedoch nicht mehr der Bibel oder der griechischen Mythologie entnommen, sondern dem, was die Historiker mit dem strittigen Begriff der >Faktengeschichte $<$ zu bezeichnen pflegen.

\section{$\mathrm{V}$. ARGENTINISCHER KONTEXT: CIVILIZACIÓN Y BARBARIE}

Humoristisch kolorierte Vielstimmigkeit, eine virtuose Mischung verschiedenster Textsorten, die intentionale Engführung des fiktionalen und des historiographischen Diskurses: Ohne Zweifel haben wesentliche Momente der palmistischen tradición Pate gestanden beim Entstehen des vorliegenden Textes. Doch welches ist die Funktion dieser peruanischen Gattung am Cono Sur? Dies war unser Thema.

»[La] ruptura del código social«, so hatte Ortega behauptet, sei das Grundgesetz der Gattung, »el gesto que es la base de la narración palmista. ${ }^{23}$ Ich möchte Gleiches von den Texten Obligados mit dieser Entschiedenheit nicht unbedingt behaupten. Dazu reicht das empirische Material,

22 Wie im Verhältnis von Maldonado-Geschichte und Exkursen, so lässt sich auch im Verhältnis der vier Exkurse zueinander ein striktes Kompositionsprinzip beobachten: Während der erste und der vierte Exkurs das Thema der menschlichen Wilden (»humanas fieras«) behandelt, erscheint im zweiten und dritten Exkurs umgekehrt das Thema der wilden Menschen (»fieras humanas«). Wir erhalten die Figur des Chiasmus.

23 Julio Ortega (1993: 428). 
über das ich verfüge, noch nicht aus, aber auf die vorliegende tradición scheint mir das Urteil Ortegas durchaus zuzutreffen. Unübersehbar formuliert »Fieras humanas « ja eine kritische Grundfrage, die den liberalen Konsens jenes Teils der argentinischen Gesellschaft, dem sich der Autor selbst verbunden fühlt ${ }^{24}$, ins Mark trifft. Fraglos handelt es sich um die erstmals von Sarmiento zum Prinzip sozialen Fortschritts erhobene Forderung nach einer kategorischen Unterscheidung zweier Kulturen ${ }^{25}$ auf argentinischem Boden: civilización y barbarie.

Vor dem Hintergrund dieser Fragestellung gewinnt dann auch die Maldonado-Geschichte wieder evaluative Relevanz. Thema der Geschichte ist nichts anderes als eine Grenzüberschreitung in mehrfacher Hinsicht. Vom Hunger getrieben - dem Naturtrieb par excellence -, setzt sich Maldonado zu Beginn über das Verbot hinweg, das Lager zu verlassen:

[...] para aplacar su hambruno, de la muy grande que por entónces diezmaba á los fundadores. (TBA, 165) ${ }^{26}$

Die Grenze der ärmlichen Zivilisation, welche die Konquistadoren errichtet haben, ist damit von Anfang an sichtbar. Maldonado überschreitet deren Grenzen und setzt sich der reinen Natur aus. Was nun allerdings geschieht, ist eine Grenzüberschreitung von erheblich größerem Gewicht: Die Erfahrung Maldonados, dass die das Lager umgebende Natur sich keinesfalls als feindliche Wildnis bzw. »Barbarei ${ }^{27}$ darbietet, sondern vielmehr als ein Lebensraum, in dem die traditionellen Werte des Humanen in ungleich besserer Weise geschützt sind als in der sogenannten Zivilisation, stellt die Grenze selbst - die Unterscheidung nämlich zwischen civilización und barbarie - in Frage. Mit dieser Erfahrung schließt Maldonado sich nicht nur selbst aus der Zivilisation, die sie verlassen hat, aus, sondern dekuvriert zugleich die latente Möglichkeit der letzteren, sich jederzeit in »Barbarei« zu verwandeln.

24 Zur Biographie Obligados cf. Antonio Pagés Larraya (1955).

25 Cf. Walter Bruno Berg (1991).

26 Auch am Ende des Schmidel-Zitates wird auf diesen Umstand nochmals hingewiesen: "La necesidad había sido causa á que desamparase á los suyos, y se metiese entre aquellos bárbaros« (TBA, 169); es sind die »indios«, die hier als »bárbaros« bezeichnet werden (ibid.).

27 Konsequent appliziert Obligado den Diskurs der »barbarie« auf die Repräsentanten der »civilización«: »Y lo demás que pasó es tan bárbaro, que no lo referimos por nuestra cuenta, y aún de repetirlo trepidáramos, si los cronistas españoles que lo confirman, no fueran tan respetables como [...] « (TBA, 168); diesem Sprachgebrauch widerspricht das Zitat in Fußnote 26 keineswegs, denn es ist ja der Chronik von Schmidel entnommen. 
Trotz der allgemeinen, genauer noch universalgeschichtlichen Thematik dieser tradición, angesichts der sogar die Ungeheuerlichkeiten von Barbarei, welche die lateinamerikanische Geschichte aufzuweisen hat, als bloße exempla erscheinen, wendet sich Obligado zugleich jedoch auch explizit an ein argentinisches Lesepublikum: Schmidels Chronik, so heißt es im ersten Satz, ist »el primer libro que sobre esta tierra se escribió« (TBA, 165). Nach dem Verlassen des Lagers folgt Maldonado dem verlassenen Ufer »de nuestro ancho río« (ibid.); schon nach kurzer Zeit überkommen sie Empfindungen bedrohlicher Einsamkeit angesichts der »inmensa Pampa« (ibid.); Ruiz Galán, der spanische Anführer, wird als »primer tirano« (ibid.) bezeichnet und Maldonado selbst schließlich als »Eva argentina, (primera mujer que menciona la Historia)« (ibid.). »Fieras humanas« ist also auch das belegen diese Zitate - eine Art argentinischer Gründungsmythos, eine Legende, die zu den Ursprüngen zurückkehrt und hier bereits auf eine Konstellation trifft, die sich im Verlauf der argentinischen Geschichte in immer neuen Varianten wiederholen wird: der Kampf um civilización und barbarie auf der einen, der Versuch, ihre Unterscheidung mit tyrannischen Mitteln zu erzwingen, auf der anderen Seite.

VI.

DIE TRADICIÓN : EIN MODERNER TEXT?

Angesichts der ideologischen Dichotomien, durch die das geistige, politische und kulturelle Klima des argentinischen Fin de siècle gekennzeichnet war, erscheint der Text im Lichte einer überraschenden Modernität. Obligado >zitiert ‘ die Parameter der zeitgenössischen Diskussion, ohne sich mit einem von ihnen vorbehaltlos zu identifizieren. So landet er mit seiner Attacke auf die zivilisatorische Grundformel des Liberalismus keineswegs beim politischen Gegner, den um die Autochthonie Argentiniens besorgten Nationalisten oder Revisionisten - also keineswegs bei einer der ideologischen Konnotationen des Gegenbegriffs barbarie -, vielmehr macht er sich zum Sprecher eines unspektakulär sanderen Zivilisationsbegriffs: Wenn die Geschichte der Gründungsfigur der argentinischen Nation mithin bereits auf eine fundamentale Ambivalenz des Begriffs der Zivilisation verweist, so kann der durch den Text postulierte sandere Zivilisationsbegriff offenbar nicht durch die Entscheidung für einen der beiden Pole der traditionellen Dichotomie verwirklicht werden, sondern deutet auf ein Kultur- 
modell, für das die Erfahrung kultureller Mehrstimmigkeit und Ambiguität $\mathrm{zu}$ bestimmenden Faktoren geworden ist. $\mathrm{Ob}$ eine solche Schlussfolgerung den expliziten Intentionen des tradicionista entsprochen hätte, ist eher fraglich. Nahegelegt wird sie jedoch durch die Textstruktur einer Gattung, die in ihrer kunstvollen Montagetechnik den ästhetischen Boden bereitet für eine Kulturerfahrung, für die ein zeitgenössischer Autor folgenden Titel gefunden hat: Argentina: la ambigüedad como destino. ${ }^{28}$

\section{Bibliographie}

\section{Literarische Werke und andere Quellen}

Obligado, Pastor S. (1898): Tradiciones de Buenos Aires, cuarta serie, con un prólogo de Ricardo Palma, Buenos Aires.

Obligado, Pastor S. (1955 [1903]): Tradiciones Argentinas, hrsg. v. Antonio Pagés Larraya, Buenos Aires: Hachette.

Palma, Ricardo (1898): „Carta-Prólogo«, in: Obligado, Pastor S.: Tradiciones de Buenos Aires, cuarta serie, con un prólogo de Ricardo Palma, Buenos Aires, pp. VIIXII.

Palma, Ricardo (1977): Cien tradiciones peruanas, hrsg. v. José Miguel Oviedo, Caracas: Biblioteca Ayacucho.

Palma, Ricardo (1993): Tradiciones Peruanas, hrsg. v. Julio Ortega, Madrid: Archivos.

Palma, Ricardo (1994): Tradiciones Peruanas, hrsg. v. Carlos Villanes Cairo, Madrid: Cátedra.

\section{Forschungsliteratur}

Berg, Walter Bruno (1991): „Civilización hecha cenizas. La presencia de Sarmiento en la novela histórica contemporánea«, in: Spiller, Roland (Hrsg.): La novela argentina de los años 80, Madrid/Frankfurt am Main: Iberoamericana/Vervuert, pp. 7797.

Berg, Walter Bruno (1996): »Rezension zu: Ricardo Szmetan, \La situación del escritor en la obra de Manuel Galván (1916-1935)«, New York 1994«, Notas 3: 2, pp. 115 117.

Berg, Walter Bruno (1998): »Identidad y alteridad en América Latina: ¿un problema de género (literario)? «, Vorlage zum $\mathrm{II}^{\circ}$ Congreso Europeo de Latinoamericanistas (4.8.9.1998), Halle; publiziert auf CD-ROM.

Berg, Walter Bruno / Schäffauer, Markus Klaus (Hrsg.) (1999): Discursos de oralidad en la literatura rioplatense del siglo XIX al XX, Tübingen: Narr.

28 Cf. Juan Carlos Chaneton (1998). 
Chaneton, Juan Carlos (1998): Argentina: la ambigüedad como destino. La identidad del país que no fue, Buenos Aires: Biblos.

Ortega, Julio (1993): »Las Tradiciones Peruanas y el proceso cultural del XIX hispanoamericano«, in: Palma, Ricardo: Tradiciones Peruanas, hrsg. v. Julio Ortega, Madrid: Archivos, pp. 409-438.

Pagés Larraya, Antonio (1955): »Pastor Obligado y las Tradiciones Argentinas «, in: Obligado, Pastor S.: Tradiciones Argentinas, hrsg. v. Antonio Pagés Larraya, Buenos Aires: Hachette, pp. 7-88.

Szmetan, Ricardo (1994): La situación del escritor en la obra de Manuel Gálvez (19161935), New York: Lang. 